\title{
Some models for immiscible displacements in Hele-Shaw cells
}

\author{
Gelu Paşa
}

\begin{abstract}
We study the linear stability of the immiscible displacement of some fluids in 2D and 3D Hele-Shaw cell. We give a method for avoiding the singularities phenomenons which appears in previous papers. In the case of a non - Newtonian fluid displaced by air in a 3D Hele-Shaw cell, we give a growth constant $\sigma$ of perturbations, which contains two new terms compared with the Saffman-Taylor formula. Our $\sigma$ has a very high growth as a parameter appearing in the constitutive relations approaches a critical value.
\end{abstract}

AMS (MOS) Subject Classification: Primary 35Q35, 76S05; Secondary: 35B20, 35B35.

\section{Introduction}

The Hele-Shaw approximation was first introduced in [6] and is concerning the flow of a fluid in the thin gap between two parallel plates. The main point is the following: the averaged (across the Hele-Shaw plates) velocities of a Stokes fluid are verifying an equation quite similar with the Darcy law for flow through a porous medium whose permeability is given in terms of the small distance between the plates.

The immiscible displacement in Hele-Shaw cells is important due to the possible applications in modeling the secondary oil recovery - see [1] and [8].

Key Words: Hele-Shaw displacement, non-Newtonian fluids, linear stability, dispersion formula

Received: December, 2016.

Revised: February, 2017.

Accepted: June, 2017. 
This method is used when the pressure of the oil in a porous medium reservoir is too low and the oil must be "pushed" by a second immiscible fluid - usually water, salt water or a polymer-water mixture. The polymer-water mixture is not a Newtonian fluid, thus the displacement of such fluids, with nonlinear constitutive relations, is an important research filed.

There exists a large literature concerning the miscible and immiscible displacement in Hele- Shaw cells - see [7] and the references therein.

In [9], [13] the authors studied the linear stability of immisicble displacement of Oldroyd-B and Maxwell type fluids by air in a 3D Hele-cell and obtained numerical values for the corresponding growth constant (in time) of the perturbations. Due to the complexity of the corresponding constitutive relations, the displacement of such fluids can not be studied in 2D Hele-Shaw cell. Moreover, the flow equations contain the derivatives of the fluid velocity, thing that does not happen in the 2D case studied in [3] and [10]. Consequently, two types of singularities appear in [9] and [13]:

a) The derivatives of the perturbed velocities become very large (tend to $\infty)$ near the basic interface between the displacing fluids;

b) The basic solution is giving us a basic pressure $P$ not depending on the variable $y$, orthogonal on the displacing direction $O x$ in the plane parallel with the cell plates. However, due to the perturbations considered in the stability analysis process, in [5] and [13] the basic pressure $P$ is depending on $y$ near the interface.

In this paper, we get a method for avoiding the previously mentioned singularities, by using some weighted amplitudes for the velocity perturbations. The displacement by air of a non-Newtonian fluid in a 3D Hele-Shaw cell is studied; we consider the effect of the meniscus curvature across the plates. The constitutive relations contain a parameter $a$, similar with the Weissenberg number in the case of an Oldroyd-B fluid. We give a rigorous proof for a formula of the growth constant $\sigma$ of the linear perturbations, in the range of small $a$. Our main conclusion is that $\sigma$ has a very high growth when the parameter $a$ appearing in the constitutive relations approaches a critical value.

We improve the results obtained in [10], where a similar formula of the growth constant was obtained, but the continuous dependence of $\sigma$ in terms of the parameter $a$ was not been rigorously obtained.

We get two results which are in contradiction with Saffman -Taylor criterion for the stability of immiscible displacement in 2D Hele-Shaw cells:

i) the displacement is almost stable when the surface tension $\gamma$ on the air-fluid interface is large enough, even if the displacing fluid is less viscous;

ii) if $\gamma=0$, then $\sigma$ tends to zero for very large wave numbers.

We give some details concerning the Hele-Shaw approximation. In our paper, the cell plates are parallel with the fixed plane $x_{1} O y$ and the distance 
between them is $b$. Two immiscible Stokes fluids with viscosities $\mu_{j}$ are displacing in the gap of thickness $b$. The velocities and pressures are denoted by

$$
\underline{\mathbf{u}}=\left(\underline{u}_{j}, \underline{v}_{j}, \underline{w}_{j}\right), \quad \underline{p}_{j}, \quad j=1,2 .
$$

The flow equations and the free-divergence condition are given below

$$
\nabla \underline{p}=\mu \Delta \underline{\mathbf{u}}, \quad \underline{u}_{j x}+\underline{v}_{j y}=0,
$$

where the lower indices $x, y$ denotes the partial derivatives.

The Hele-Shaw approximation is based on the following hypothesis:

$H 1: \underline{u}_{j x}, \underline{u}_{j y}, \underline{v}_{j x}, \underline{v}_{j y}, \underline{w}_{j} \approx 0, j=1,2$. Then the flow equations become

$$
\underline{p}_{j x}=\mu \underline{u}_{j z z}, \quad \underline{p}_{j y}=\mu \underline{v}_{j z z}, \quad \underline{p}_{j z}=0 .
$$

We average the velocities across the plates and get

$$
<\underline{\mathbf{u}}_{\mathbf{j}}>:=\frac{1}{b} \int_{0}^{b} \underline{\mathbf{u}}_{\mathbf{j}}(\mathbf{z}) d z=-\frac{b^{2}}{12 \mu_{j}} \nabla \underline{p}_{j}=-\left(k / \mu_{j}\right) \nabla \underline{p}_{j} .
$$

The above equations are quite similar to Darcy law for flow in a porous medium with permeability $k=b^{2} / 12$.

$H 2$ : There is a sharp interface between the two immiscible fluids, where Laplace law is assumed; this means: i)the pressure jump is given by the surface tension multiplied with the interface curvature; ii) the normal velocity across the interface is continuous.

\section{Two Stokes fluids displacing in a 2D Hele-Shaw}

We first recall the result given in [10]. Consider the fluid 1 is displacing fluid 2 ; for both fluids we have

$$
\underline{\mathbf{u}_{\mathbf{j}}}=\left(\underline{u}_{j}, \underline{v}_{j}\right)=-\left(k / \mu_{j}\right) \nabla \underline{p}_{j}=\nabla \underline{\phi}_{j} ; \quad \Delta \underline{\phi}_{j}=0 ; \quad j=1,2 ; \quad k=b^{2} / 12 .
$$

Here $\underline{u}_{j}, \underline{v}_{j}$ are the filtration (averaged) velocities.

The basic flow is given by the velocity $(V, 0)$ of the fluid 1 far upstream, the basic interface is $x=0$ in the moving frame $x=x_{1}-V t$. We consider the surface tension $T$ on $x=0$. We also assume Laplace's law near the basic interface. We emphasize that the flow equations do not contain $\underline{u}_{j x}, \underline{u}_{j y}, \underline{v}_{j x}, \underline{v}_{j y}$.

Saffman and Taylor [12] assumed the following small perturbation of the basic interface $x=0$ :

$$
x=c \exp (i n y+\sigma t), \quad n=\text { wave numbers }, \quad \sigma=\text { growth rate. }
$$


The perturbations are denoted by $p,(u, v), \phi$. We have $\Delta \phi=0$. As the normal component of the velocity is continuous and the interface is material, the following equation "to the first order of deviation" was considered in [12] near $x=0$ :

$$
\phi_{1 x}=\phi_{2 x}=V+c \sigma \exp (i n y+\sigma t)
$$

and the following solution was obtained

$$
\left\{\begin{array}{l}
\phi_{1}=V x+(c \sigma / n) \exp (i n y+n x+\sigma t), x<0 \\
\phi_{2}=V x-(c \sigma / n) \exp (i n y-n x+\sigma t), x>0
\end{array}\right.
$$

We have two remarks concerning the solution (4):

Remark 1. The perturbed interface is material, then it follows (we omit the indices $j=1,2)$ :

$$
x_{t}=u, \quad u=c \sigma \exp (\text { iny }+\sigma t) \Rightarrow u=u(y, t) .
$$

As $u_{x}+v_{y}=0$, we get $v_{y}=0$, then from (1) it follows $p_{x}=p_{x}(y, t), \quad p_{y}=$ $p_{y}(x, t)$. Moreover, we need $p_{x y}=p_{y x}$, therefore we obtain (for both fluids)

$u_{y}=v_{x}=A=$ constant $, \quad u=A y+B, \quad v=A x+C \quad$ with $\quad B, C=$ constant.

We consider that this dependence of $u, v$ in terms of $x, y$ is not acceptable from physical point of view.

Remark 2. Solution (4) verifies the equation (3) only if for small $|x|$ (near the basic interface) we have

$$
x>0, \quad \exp (-n x) \approx 1 \quad \text { and } \quad x<0, \quad \exp (n x) \approx 1 .
$$

Even if $x$ is very small, for large values of the wave number $n$ we have

$$
x>0, \quad \exp (-n x) \approx 0 \quad \text { and } \quad x<0, \quad \exp (n x) \approx 0 .
$$

Then for small $|x|$ and large $n$, the solution (4) is giving $\phi_{1}=\phi_{2}=V x$ and the equation (3) is not verified.

According to Saffman and Taylor [12] , the pressures are obtained from the potential expressions (1). The Laplace law on the interface is

$$
p_{2}-p_{1}=T x_{y y}
$$


then we get

$$
\frac{\sigma}{n}\left\{\frac{\mu_{2}}{k} \exp (-n x)+\frac{\mu_{1}}{k} \exp (n x)\right\}=V\left\{\frac{\mu_{2}}{k}-\frac{\mu_{1}}{k}\right\}+T\left(-n^{2}\right) .
$$

We can now emphasize that Saffman- Taylor formula (6) holds only if $\exp \left({ }_{-}^{+} n x\right) \approx$ 1 (see Remark 2)

$$
\sigma_{S T}=\frac{V n\left(\mu_{2}-\mu_{1}\right)-\left(b^{2} / 12\right) T n^{3}}{\mu_{2}+\mu_{1}} .
$$

The well known Saffman-Taylor instability criterion was obtained from this last relation:

$$
\mu_{1}<\mu_{2} \Rightarrow \max _{n}\left[\sigma_{S T}\right]>0 .
$$

For the case when the displacing fluid is air, we have $\mu_{1} \approx 0$, therefore the above growth constant (6) becomes

$$
\sigma_{S T-A I R}=V n-\left(b^{2} / 12\right)\left(T / \mu_{2}\right) n^{3} .
$$

We now recall the result of Gorell and Homsy [5]. The basic velocity and pressure are denoted by $(V, 0), P$ and we have

$$
P_{j x}=-\left(\mu_{j} / k_{j}\right) V, \quad P_{j y}=0,
$$

therefore $P$ is depending only on $x$ - this is an important property. Moreover, as the basic interface is the straight line $x=0$, the basic pressure $P$ is continuous at $x=0$. As before, the perturbations are denoted by $u, v, p$. The following Fourier decomposition is used for the perturbed velocity $u$ :

$$
u=f(x) \exp (\sigma t) \cos (n y) .
$$

The free divergence condition gives us the solution for $v$ :

$$
v=(-1 / n) f_{x} \exp (\sigma t) \sin (n y) .
$$

Since the perturbed Darcy law gives us $p_{j y}=-\left(\mu_{j} / k_{j}\right) v$ we easily get the expression of the perturbed pressure in both fluids:

$$
p_{j}=-\left(\mu_{j} / k_{j} n^{2}\right) f_{x} \exp (\sigma t) \cos (n y), \quad j=1,2 .
$$

Cross derivation of the pressure gives us the equation for the amplitude $f(x)$ :

$$
f_{x x}-n^{2} f=0 .
$$


As $u$ is continuous at $x=0$ and the perturbations must decay to zero far away from $x=0$, Gorell and Homsy [5] considered the following solutions for the amplitude:

$$
f(x)=f(0) \cdot\left\{\begin{array}{cc}
\exp (n x), & x \leq 0 \\
\exp (-n x), & x \geq 0
\end{array} ;\left\{\begin{array}{c}
f_{x}^{-}(0)=n f(0) \\
f_{x}^{+}(0)=-n f(0) .
\end{array}\right.\right.
$$

Here the dimension of $f(0)$ is unit of length over unit of time.

The perturbed interface is denoted by $\eta$; we have $\eta_{t}=u$ and thus

$$
\eta(x, y, t)=(1 / \sigma) f(x) \exp (\sigma t) \cos (n y)
$$

The limit values of the pressure near the basic interface were obtained in [5] by considering a first order Taylor expansion of the basic pressure and the perturbed pressure (11):

$$
\begin{gathered}
p^{+}(0)=P(0)+P_{x}^{+}(0) \eta(0)+p_{2}(0)= \\
P(0)-\frac{\mu_{2}}{k_{2}} V \frac{1}{\sigma} f(0) \exp (\sigma t) \cos (n y)-\frac{\mu_{2}}{k_{2} n^{2}} f_{x}^{+}(0) \exp (\sigma t) \cos (n y), \\
p^{-}(0)=P(0)+P_{x}^{-}(0) \eta(0)+p_{1}(0)= \\
P(0)-\frac{\mu_{1}}{k_{1}} V \frac{1}{\sigma} f(0) \exp (\sigma t) \cos (n y)-\frac{\mu_{1}}{k_{1} n^{2}} f_{x}^{-}(0) \exp (\sigma t) \cos (n y) .
\end{gathered}
$$

From (12), (13), (14) we recover the formula (6).

Gorell and Homsy's model [5] uses the perturbations of the velocities and not a perturbation of the basic interfaces. Moreover, the free-divergence condition and then the partial derivatives $u_{x}, v_{y}$ were used in their paper.

Remark 3. The modulus of $u_{x}, v_{y}$ becomes very large for $x \approx 0$ and $n \rightarrow \infty$. Indeed, $u_{x}, v_{y}$ contain the factor $[-n \exp (-n x)]$. Consider $n$ as a real (and not integer) number, $x$ as a parameter, and the function

$$
F(n)=-n \exp (-n x) .
$$

We use the derivative $F_{n}$ of $F$ in terms of $n$ and get

$F_{n}(n=1 / x)=0, F_{\text {min }}=F_{\text {min }}(x)=F(1 / x)=-1 /(e x), F_{\text {min }}(x \rightarrow 0) \rightarrow-\infty$.

To overcome the phenomenon described in the above remark, we consider the new expansion

$$
u=\exp (-n[\alpha+x]+\sigma t) \cos (n y), \quad \alpha>0 .
$$


Therefore, in the point $x=0$, the derivatives of the velocity contain the factor

$$
F A(n)=-n \exp (-n \alpha)
$$

This time we have

$$
\min _{n}[-n \exp (-n \alpha)] \rightarrow 0 \quad \text { for } \quad \alpha \rightarrow \infty
$$

We consider two particular cases:

$\max _{n}|F A(n)| \approx 370$ for $\quad \alpha=0.001 ; \max _{n}|F A(n)| \approx 0.0075$ for $\alpha=50$.

Remark 4. The basic pressure at the "point" $\eta$ is depending on $y$ which contradicts the relation (8). Indeed, if we use a first Taylor expansion of $P$ near $x=0$, as in (13), the Fourier expansion (9) gives us

$$
P_{y}^{+}(\eta) \approx P_{x}^{+}(0)(-n) \frac{f(0)}{\sigma} \exp (\sigma t) \sin (n y) \neq 0 .
$$

We use (15) instead of (9) and get

$$
P_{y}^{+}(\eta) \approx P_{x}^{+}(0)[(-n) \exp (-n \alpha)] \frac{f(0)}{\sigma} \exp (\sigma t) \sin (n y) .
$$

As we pointed out in $\operatorname{Remark} 3, \max _{n}\{|n \exp (-n \alpha)|$ is very small for large $\alpha$. Therefore $P_{y}(\eta)$ is very close to zero for large $\alpha$. Then we can consider that the dependence of $P$ in terms of $y$ is arbitrary small for large enough $\alpha$.

\section{A non-Newtonian fluid displaced by air}

Consider the non-Newtonian fluid governed by the following constitutive relations:

$$
\underline{\tau}=\mu \underline{D}+\mu a\left(\underline{L D}+\underline{D L}^{T}\right), \quad a>0,
$$

where the dimension of $a$ is (time). Here we use the following notations:

$\underline{\tau}, \underline{D}$ are the the extra-stress and strain-rate tensors; $\mu$ is the fluid viscosity; $\underline{L}$ is the matrix of the velocity gradients. We have the relations

$$
\underline{L}_{i j}=\partial \underline{u}_{i} / \partial x_{j}, \quad\left(\underline{L}_{i j}\right)^{T}=\underline{L}_{j i}, \quad \underline{D}=\left(\underline{L}+\underline{L}^{T}\right) .
$$

Our constitutive relations are steady; it can be proved that (16) are frameindependent with respect to the coordinate changes $x^{+}=Q x$, where $Q$ is 
an ortonormal matrix not depending on time. We consider an incompressible fluid, then we have

$$
\underline{u}_{x}+\underline{v}_{y}+\underline{w}_{z}=0
$$

The no-slip conditions on the plates are imposed for the velocity:

$$
(\underline{u}, \underline{v}, \underline{w})=0 \quad \text { at } \quad z=0, \quad z=b .
$$

The flow equations of our fluid are given below:

$\underline{p}_{x}=\underline{\tau}_{11, x}+\underline{\tau}_{12, y}+\underline{\tau}_{13, z} ; \quad \underline{p}_{y}=\underline{\tau}_{21, x}+\underline{\tau}_{22, y}+\underline{\tau}_{23, z} ; \quad \underline{p}_{z}=\underline{\tau}_{31, x}+\underline{\tau}_{32, y}+\underline{\tau}_{33, z}$.

We consider the following basic flow (in the positive direction of $x$-axis) denoted by the super-index ${ }^{0}$ :

$$
\begin{gathered}
\nabla p^{0}=\left(p_{x}^{0}(x), 0,0\right), \quad \mathbf{u}^{0}=\left(u^{0}(z), 0,0\right), \\
L_{i j}^{0}=0 \quad \forall \quad(i, j) \neq(1,3), \quad L_{13}^{0}=u_{z}^{0}, \\
D_{i j}^{0}=0 \quad \forall \quad(i, j) \neq(1,3) \text { and }(3,1), \quad D_{13}^{0}=D_{31}^{0}=u_{z}^{0}, \\
\tau^{0}=\mu D^{0}+a \mu\left\{L^{0} D^{0}+D^{0} L^{0 T}\right\} .
\end{gathered}
$$

Then we get

$$
\begin{gathered}
\tau^{0}=\mu\left(\begin{array}{ccc}
0 & 0 & u_{z}^{0} \\
0 & 0 & 0 \\
u_{z}^{0} & 0 & 0
\end{array}\right)+a \mu\left(\begin{array}{ccc}
2\left(u_{z}^{0}\right)^{2} & 0 & 0 \\
0 & 0 & 0 \\
0 & 0 & 0
\end{array}\right), \\
\tau_{22}^{0}=\tau_{23}^{0}=\tau_{33}^{0}=\tau_{12}^{0}=0, \quad \tau_{11}^{0}=2 a \mu\left(u_{z}^{0}\right)^{2}, \quad \tau_{13}^{0}=\mu u_{z}^{0} .
\end{gathered}
$$

We have the following basic flow equations:

$$
\begin{aligned}
& p_{x}^{0}=\tau_{11, x}^{0}+\tau_{12, y}^{0}+\tau_{13, z}^{0}, \\
& p_{y}^{0}=\tau_{21, x}^{0}+\tau_{22, y}^{0}+\tau_{23, z}^{0}, \\
& p_{z}^{0}=\tau_{31, x}^{0}+\tau_{32, y}^{0}+\tau_{33, z}^{0},
\end{aligned}
$$

and thus $p_{y}^{0}=p_{z}^{0}=0$. Moreover, since $\tau_{11}^{0}=\tau_{11}^{0}(z)$, from the equation (26) it follows

$$
p_{x}^{0}(x)=\tau_{13, z}^{0}(z)=\mu u_{z z}^{0}=G=\text { constant }<0,
$$

because the pressure is decreasing in terms of $x$. The fluid displacement is produced by the pressure gradient $G$, which is giving the basic flow with the 
velocity $\left(u^{0}(z), 0,0\right)$. From $(29)$ we get the component $u^{0}$ of the basic velocity in terms of $G$

$$
u^{0}=\frac{G}{2 \mu}\left(z^{2}-b z\right)
$$

The characteristic velocity $U$ of our basic flow is given by

$$
U=<u^{0}>:=\frac{1}{b} \int_{0}^{b} u^{0}(z) d z=-\left(\frac{b^{2}}{12 \mu}\right) G .
$$

We use $U$ and introduce the dimensionless Weissenberg number $W$ :

$$
W=a U / l,
$$

where $l$ is the characteristic length of our Hele-Shaw cell.

In the following we perform the modal linear stability of the above basic flow (21)- (22) in the range of small $W$ of order $\epsilon=b / l$.

The relation (31) is quite similar with Darcy law for the flow through a porous media with filtration velocity $<u^{0}>$ and "permeability" $b^{2} / 12$.

In [13], it is (numerically) studied the displacement of an Oldroyd-B fluid by air in a 3D Hele-Shaw cell. It was supposed that the pressure can depend on time - that means in the pressure expression we can add a constant not depending on $x$. As in [13], we consider the following dependence of the pressure in terms of the time $t$ (which first appears here):

$$
p^{0}=G\left(x-<u^{0}>t\right), \quad \text { for } \quad x><u^{0}>t .
$$

The basic moving interface between air and our fluid is

$$
x=<u^{0}>t .
$$

The perturbations of the basic flow are denoted by $(u, v, w), p, \tau$.

We recall the free-divergence condition (18). We suppose $u=v=w=0$ on the Hele-Shaw plates, then the velocity perturbations verify the relation

$$
\int_{0}^{b}\left(u_{x}+v_{y}\right)=0 .
$$

A solution for the above equation is $u_{x}+v_{y}=0$, then we get $w_{z}=0$ and the boundary conditions are giving us $w=0$. In the following we shall consider

$$
u_{x}+v_{y}=0, \quad w=0 .
$$

Equation (26) may have other solutions, but in this paper we only consider the solution (37). 
As $w=0$, the perturbations $L, D$ of $L^{0}, D^{0}$ are given by

$$
L=\left(\begin{array}{ccc}
u_{x} & u_{y} & u_{z} \\
v_{x} & v_{y} & v_{z} \\
0 & 0 & 0
\end{array}\right), \quad D=\left(\begin{array}{ccc}
2 u_{x} & u_{y}+v_{x} & u_{z} \\
u_{y}+v_{x} & 2 v_{y} & v_{z} \\
u_{z} & v_{z} & 0
\end{array}\right)
$$

Inserting them in (16) and using (24), in the frame of the linear stability analysis, we get

$$
\tau=\mu\left\{D+a\left[L^{0} D+\left(L^{0} D\right)^{T}+L D^{0}+\left(L D^{0}\right)^{T}\right]\right\} .
$$

The components of the extra-stress tensor in terms of the velocity perturbations are given by the relations

$$
\begin{gathered}
\tau_{11}=2 \mu u_{x}+4 a \mu u_{z}^{0} u_{z}, \quad \tau_{12}=\mu\left(u_{y}+v_{x}\right)+2 a \mu u_{z}^{0} v_{z}, \\
\tau_{13}=\mu u_{z}+a \mu u_{z}^{0} u_{x}, \quad \tau_{22}=2 \mu v_{y}, \quad \tau_{23}=\mu v_{z}+a \mu u_{z}^{0} v_{x}, \quad \tau_{33}=0 .
\end{gathered}
$$

It is interesting to see that the relations (39) - (40) can be also obtained directly from the relations (25). The corresponding pressure perturbations are

$p_{x}=\tau_{11, x}+\tau_{12, y}+\tau_{13, z}, \quad p_{y}=\tau_{21, x}+\tau_{22, y}+\tau_{23, z}, \quad p_{z}=\tau_{31, x}+\tau_{32, y}+\tau_{33, z}$

We use (30) and consider the following Fourier decomposition for $(u, v)$ :

$$
\begin{gathered}
u=f(z) \exp (-n[\alpha+x]+\sigma t) \cos (n y), \\
v=f(z) \exp (-n[\alpha+x]+\sigma t) \sin (n y), \\
f(z)=\frac{1}{n l} \cdot \frac{W}{504} \cdot \frac{G}{2 \mu} z(z-b)=\frac{1}{n l} \cdot \frac{W}{504} u^{0}, \quad x \geq 0, \quad n \geq 1 .
\end{gathered}
$$

where $\sigma$ is the growth constant and $\alpha$ is a large positive number. The above expansion (42) verifies the relations $u_{x}+v_{y}=0$ and $u=v=0$ for $z=0, z=b$. Moreover, it follows

$$
\begin{gathered}
u_{x}=-n u, \quad u_{y}=-n v, \quad v_{x}=-n v, \quad u_{x y}=\left(n^{2}\right) v, \quad v_{x y}=\left(-n^{2}\right) u, \\
u_{x x}+u_{y y}=0, \quad u_{z x}+v_{z y}=0, \quad u_{x x}+v_{x y}=0 .
\end{gathered}
$$

Equations (39) - (44) give us the pressure perturbations in terms of the velocity perturbations:

$$
p_{x}=a \mu \cdot\left(3 u_{z}^{0} u_{z x}+u_{z z}^{0} u_{x}\right)+\mu u_{z z}, \quad p_{y}=a \mu \cdot\left(3 u_{z}^{0} v_{z x}+u_{z z}^{0} v_{x}\right)+\mu v_{z z} .
$$

and we obtain $p_{x y}=p_{y x}$. We get also

$$
p_{z}=\mu\left(u_{z}+a u_{x} u_{z}^{0}\right)_{x}+\mu\left(v_{z}+a v_{x} u_{z}^{0}\right)_{y}=0 \Rightarrow
$$




$$
\left(p_{x}\right)_{z}=a \mu \cdot\left(4 u_{z z}^{0} u_{z}+3 u_{z}^{0} u_{z z}\right)_{x}+\mu u_{z z z}=0 .
$$

The following dimensionless quantities will be used in this section:

$$
\begin{gathered}
x^{\prime}=\frac{x}{l}, \quad y^{\prime}=\frac{y}{l}, \quad z^{\prime}=\frac{z}{b}, \quad\left(u^{\prime}, v^{\prime}\right)=\frac{(u, v)}{U}, \quad \epsilon=\frac{b}{l}<<1 . \\
p^{\prime}=p \frac{1}{G l}, \quad t^{\prime}=t \frac{U}{l}, \quad \sigma^{\prime}=\sigma \frac{l}{U} ; \quad f^{\prime}=f / U ; \quad \gamma^{\prime}=\gamma \frac{1}{\mu U} ; \quad n^{\prime}=n l .
\end{gathered}
$$

Recall (30) and (31). We have

$$
\begin{gathered}
u^{0}=-\frac{12 \mu U}{2 b^{2} \mu} b^{2} z^{\prime}\left(z^{\prime}-1\right)=-6 U z^{\prime}\left(z^{\prime}-1\right) \Rightarrow \\
u^{0^{\prime}}=-6 z^{\prime}\left(z^{\prime}-1\right), \quad f^{\prime}=-6\left(1 / n^{\prime}\right)(W / 504) z^{\prime}\left(z^{\prime}-1\right) .
\end{gathered}
$$

We use the above last expression to obtain the dimensionless form of the relation (47). As $f_{z^{\prime} z^{\prime} z^{\prime}}^{\prime}=0$, we have

$$
L H S:=\frac{W}{504} a\left[4 \cdot 12 U \cdot 6 U\left(2 z^{\prime}-1\right)+3 \cdot 6 U\left(2 z^{\prime}-1\right) 12 U\right] \frac{1}{l}=0 .
$$

In this section we consider $W=O(\epsilon), \quad \epsilon=O\left(10^{-3}\right)$.

Recall $W=a U / l$. As $L H S=(W / 504) W \cdot 504\left(2 z^{\prime}-1\right) \leq W^{2}$, we can see that (51) is not verified exactly, but with the precision order $O\left(\epsilon^{2}\right)$. Then the amplitude $f$ given by (42) verifies the equation (51) with the precision order $O\left(\epsilon^{2}\right)$.

We follow Wilson [13] and consider the kinetic and dynamic boundary conditions on the steady air-liquid interface $x=<u^{0}>t$. The perturbed interface is given by

$$
\psi=x-<u^{0}>t
$$

As the interface is material, we also have

$$
\psi_{t}=u \Rightarrow \psi=u / \sigma
$$

The stress jump on the interface is given by the surface tension multiplied with the interface curvature (Laplace's law). We consider

$$
<G \psi+p-\tau_{11}>=\gamma \cdot\left\langle\left\{\psi_{y y}+\psi_{z z}\right\}>\right.
$$

where $\gamma$ is the surface tension and $\left\{\psi_{y y}+\psi_{z z}\right\}$ is denoting the total curvature of the interface. A quite similar formula is used in [13], but without the term containing $\psi_{z z}$. 
In many papers, the term $\tau_{11}$ did not appears in Laplace's law (54). On the contrary, the importance of this term is rigorously proved in [2] and [11] - see also the references therein.

The partial derivative with respect to $x$ is equivalent with the multiplication with $(-n)$. The relations $(45)$ and $(39)-(40)$ are used to obtain $\left(p-\tau_{11}\right)$ :

$$
\begin{gathered}
p_{x}-\tau_{11, x}=\mu\left(-2 n^{2} u\right)+a \mu n\left(u_{z}^{0} u_{z}-u_{z z}^{0} u\right)+\mu u_{z z} \Rightarrow \\
p-\tau_{11}=(-1 / n)\left\{\mu\left(-2 n^{2} u\right)+\operatorname{a\mu n}\left(u_{z}^{0} u_{z}-u_{z z}^{0} u\right)+\mu u_{z z}\right\} .
\end{gathered}
$$

From the equations (53) and (42) it follows the expressions of $\psi_{x}, \psi_{y y}, \psi_{y y}$. We insert them and the expression (55) in the relation (54) to get

$<\frac{G(-n) f}{\sigma}-2 n^{2} \mu f+a \mu n \cdot\left(u_{z}^{0} f_{z}-u_{z z}^{0} f\right)+\mu f_{z z}>=\frac{\gamma}{\sigma}<-n^{2} f+f_{z z}>(-n)$.

We perform the average across the plates and it follows the dispersion relation

$$
\sigma=\frac{U n-(\gamma / \eta)\left(b^{2} / 12\right) n^{3}-(\gamma / \eta) n}{1-2 a U n+b^{2} n^{2} / 6}
$$

Recall the growth rate obtained in [12] for a Newtonian liquid with viscosity $\mu$ displaced by air in a 2D Hele-Shaw cell:

$$
\sigma_{S T}=U n-(\gamma / \mu)\left(b^{2} / 12\right) n^{3}
$$

Remark 5. The same method as in section 3 was used in the talks [3] and [4], obtained in collaboration with professor Prabir Daripa from Texas A-M University, College Station, USA. In these talks was studied the linear instability of the displacement by air of an Oldroyd-B fluid (with more complex constitutive relations) in a Hele-Shaw cell, for small Weissenberg numbers. A different Fourier expansions and amplitudes of the velocity perturbations were used, giving unbounded partial derivatives of $(u, v)$ with respect to $x, y$ near the basic interface $x=0$ - as we pointed out in Remark 3. Despite this fact, a quite similar formula for the growth constant and a strong destabilizing effect (compared with the case studied in [10]) were obtained. Unfortunately, both these talks have not been published.

\section{Conclusions}

1) For the case $a=0$, the formula (57) of $\sigma$ is quite similar with the SaffmanTaylor growth constant (58), but two new terms appear:

a) $(-\gamma / \mu) n$ in the numerator, given by the meniscus curvature; 
b) $b^{2} n^{2} / 6$ in the denominator, given by the partial derivative with respect to $x$ of the velocity.

We use the dimensionless quantities (48) - (49), then from (57) we get the following dimensionless approximate formula of the growth constant for small $W$, denoted by $\sigma_{S W}^{\prime}$ :

$$
\sigma_{S W}^{\prime}=\frac{n^{\prime}-\gamma^{\prime} n^{\prime}-\gamma^{\prime}\left(\epsilon^{2} / 12\right) n^{\prime 3}}{1-2 W n^{\prime}+\epsilon^{2} n^{\prime 2} / 6}
$$

The dimensionless quantities and the formula (58) give us the dimensionless Saffman-Taylor growth constant

$$
\sigma_{S T}^{\prime}=n^{\prime}-\gamma^{\prime}\left(\epsilon^{2} / 12\right) n^{\prime 3}
$$

2) Consider $W=c \epsilon$ with $c>0.40824$. Then the denominator of (59) has two real roots. Indeed, we have

$$
\Delta=c^{2} \epsilon^{2}-\epsilon^{2} / 6>0 \Leftrightarrow c^{2}>1 / 6, \quad c>0.40824 .
$$

3) In the range $W=c \epsilon$ with $c^{2}<1 / 6$, the flow is almost stable when $\gamma^{\prime}>1$, even if the displacing fluid is less viscous (air). Indeed, in this case the denominator of (59) is positive $\forall n^{\prime}$ and we have

$$
\sigma_{S W}^{\prime}<\frac{n^{\prime}\left(1-\gamma^{\prime}\right)}{1-2 W n^{\prime}+\epsilon^{2} n^{\prime 2} / 6} .
$$

Moreover, we can see that in the case $\gamma^{\prime}=0$, we have

$$
\lim _{n \rightarrow \infty} \sigma_{S W}^{\prime}=0 .
$$

The last two relations are in contradiction with the Saffman-Taylor stability critertion.

In Figure 1 we plot the positive growth constant (60) (on the vertical axis) in terms of the wave number $n^{\prime}$ (on the horizontal axis), when $\epsilon=0.006, \quad \gamma^{\prime}=$ $0.1, \quad W=c \epsilon$ and

$c=0.1$ (lower curve), 0.2, 0.3, 0.35. 0,38, 0.39, 0.395, 0.4, 0.405 (upper curve).

We can see that $\sigma_{S W}^{\prime}$ is increasing from 8000 to over 18000 when $c$ is increasing from 0.4 to 0.405 . We must avoid the value $c=0.40824$ where a blow-up of the growth constant $\sigma_{S W}^{\prime}$ appears. On the other hand, we use (60) and get

$$
\max _{n^{\prime}}\left\{\sigma_{S T}^{\prime}\right\} \approx 1.000 \text {. }
$$




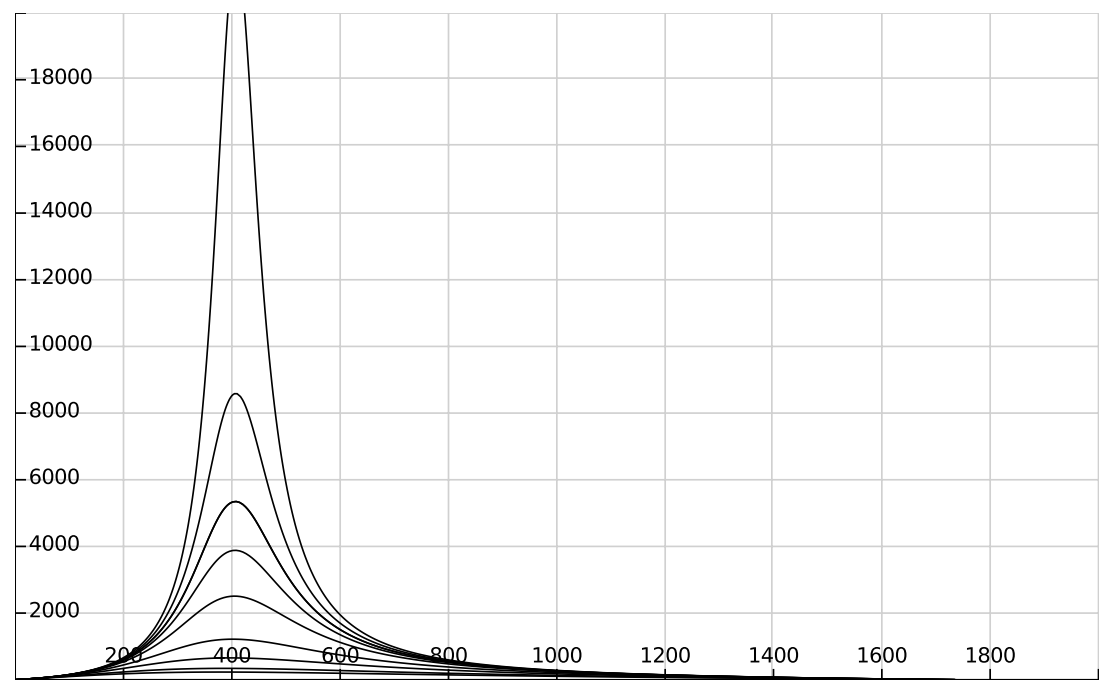

Figure 1: The growth constant (59) for $\epsilon=0.006, \gamma^{\prime}=0.1, W=c \epsilon$ and $c=0.1,0.2,0.3,0.35 .0,38,0.39,0.395,0.4,0.405$.

\section{References}

[1] J. BeAr, Dynamics of Fluids in Porous Media, Elsevier, New York, 1972.

[2] J. M. Bush, 2013, Surface Tension Module, Lect. Notes, MIT.

[3] P. Daripa and G. Paşa, Saffman-Taylor instability for an Oldroyd-B fluid, Workshop "Enhanced Oil Recovery and Porous media Flows", July 31 August 1, 2013, Doha, Qatar.

[4] P. Daripa and G. Paşa, Saffman-Taylor Instability for a non-Newtonian fluid, 66th Annual Meeting of the APS Division of Fluid Dynamics, Session M30, Nov. 26, 2013, Pittsburgh, Pennsylvania, USA. 
[5] S. B. Gorell and G. M. Homsy, 1983, A theory of the optimal policy of oil recovery by secondary displacement process, SIAM J. Appl. Math. 43(1), 79 .

[6] H. S. Hele-Shaw, 1898, Investigations of the nature of surface resistence of water and of streamline motion under certain experimental conditions, Inst. Naval Architects, Transactions, 40, 21-46.

[7] R. Krechetnikov and G. M.Homsy, 2004, On a new surfactant-driven fingering phenomenon in a Hele-Shaw cell, J. Fl. Mech., 500, 103-124.

[8] H. Lamb, Hydrodynamics, Cambridge University Press, Cambridge, 1933.

[9] S. Mora and M. Manna, 2009, Saffman-Taylor instability for generalized newtonian fluids, Phys. Rev. E, 80, pp. 016308.

[10] G. Pasa, Some non-Newtonain effects in Hele-Shaw displacement, 2016, Rev. Roumaine Math. Pures Appl., 61(4), 293-304.

[11] M. Renardy and Yuriko Renardy, 1991, On the nature of boundary conditions for flows with moving free surfaces, J. Comput. Physics, 93, 325-335.

[12] P. G. Saffman and G. I. Taylor, 1958, The penetration of a liquid into a porous medium of Hele-Shaw cell containing a more viscous fluid, Proc. Roy. Soc. Lond., A 245, 312-329.

[13] S. D. R. Wilson, The Taylor-Saffman problem for a non-Newtonian liquid, 1990, J. Fl. Mech., 220, 413-425.

Gelu PAŞA,

"Simion Stoilow" Institute of Mathematics of the Ro-

manian Academy,

Calea Grivitei 21, Bucharest S1, Romania,

e-mail: gelu.pasa@imar.ro 